\title{
Positron Induced Fusion Pulsed Space Propulsion through an Ultra-Intense Laser
}

\author{
Robert LeMoyne \\ Independent (Senior Member AIAA \& Senior Member IEEE), Running Springs, CA, USA \\ Email: rlemoyne07@gmail.com
}

How to cite this paper: LeMoyne, R. (2017) Positron Induced Fusion Pulsed Space Propulsion through an Ultra-Intense Laser. Journal of Applied Mathematics and Physics, 5, 813-821. https://doi.org/10.4236/jamp.2017.54070

Received: September 28, 2016

Accepted: April 9, 2017

Published: April 12, 2017

\begin{abstract}
A pulsed space propulsion system using position antimatter to induce Deuterium-Tritium fusion through an ultra-intense laser incident on a gold target is conceptually presented through fundamental performance analysis. As opposed to traditional strategies positron antimatter is considered rather than antiproton antimatter. Positron antimatter can be produced by an ultraintense laser incident on a high atomic number target, such as gold. The ultra-intense laser production of positron antimatter mechanism greatly alleviates constraints, such as requirements for antimatter storage imperative for antiproton antimatter. Also the ultra-intense laser and associated energy source can be stationary or positioned remote while the pulsed space propulsion system using position antimatter to induce Deuterium-Tritium fusion is in flight. Various mechanisms for antimatter catalyzed fusion are considered, for which the preferred mechanism is the antiproton hotspot ignition strategy. Fundamental performance analysis is subsequently applied to derive positron antimatter generation requirements and associated propulsion performance. The characteristics of the pulsed space propulsion system using position antimatter to induce Deuterium-Tritium fusion through an ultra-intense laser incident on a gold target imply a promising non-chemical propulsion alternative for the transport of bulk cargo to support space missions.
\end{abstract}

\section{Keywords}

Antimatter Induced Fusion, Pulsed Space Propulsion, Nuclear Fusion, Ultra-Intense Laser, Bethe-Heitler Process, Positron, Antiproton Hotspot Ignition

\section{Introduction}

The recent capability of ultra-intense lasers has enabled new strategies for nonchemical propulsion systems. For example, ultra-intense lasers have demon- 
strated the capacity to generate positron antimatter while incident on a high atomic number target, such as gold. LeMoyne and Mastroianni conceptually demonstrated an approach to achieving pulsed antimatter propulsion through the production of positron antimatter by means of an ultra-intense laser striking a gold target. A notable aspect of the proposed ultra-intense laser positron antimatter pulsed propulsion system is the decoupling of the propulsion apparatus and laser energy source proximity, for which the energy source of the laser can be ground based while the propulsion system is in flight [1] [2] [3] [4].

Another non-chemical propulsion system incorporates photofission as a propulsive energy source. Photofission is resultant of ultra-intense laser energy incident on a high atomic number target, such as natural lead or uranium-238, which is a predominant isotope of uranium. Using a pulsed propulsion configuration LeMoyne and Mastroianni proposed a pulsed propulsion system incorporating photofission as the energy source for the propulsive fluid. An advantage of photofission is the use of naturally abundant target sources, such as natural lead or uranium-238 [5].

Further observation of the characteristics were manifested in Project New Orion as advocated by LeMoyne and Mastroianni. In contrast to the original Project Orion, which advocated a thermonuclear detonation of considerable magnitude, Project New Orion elucidated a more feasible perspective with regards to testing and evaluation. Using advanced energy conversion techniques, such as magneto-hydrodynamic generation, to provide an energy source for the ultra-intense laser, the system demonstrated the capacity to impart a kinetic energy impulse on a launch vehicle upper stage [6].

Fission enables realizable pathways to non-chemical nuclear propulsion, such as conceptual designs for the NERVA rocket and Project Orion [7] [8] [9]. A representative fission reaction is demonstrated in Equation (1). However, issues can arise, such as the side effects of the fission byproducts and complex mechanisms for regulating the inherent neutron flux [5] [6]. Another serious consideration involves fission byproducts, such as strontium-89, which is chemically analogous to calcium [10].

$$
\begin{aligned}
& \text { Fission : }{ }_{0}^{1} n+{ }_{92}^{235} \mathrm{U} \rightarrow{ }_{56}^{141} \mathrm{Ba}+{ }_{36}^{92} \mathrm{Kr}+3{ }_{0}^{1} n \\
& \text { Fusion }:{ }_{1}^{2} \mathrm{H}+{ }_{1}^{3} \mathrm{H} \rightarrow{ }_{2}^{4} \mathrm{He}+{ }_{0}^{1} n \quad[10]
\end{aligned}
$$

Another mechanism for a nuclear reaction is fusion. Fusion offers a superior specific energy yield to fission. The byproducts of a fusion reaction are relatively cleaner by contrast to fission [10]. Equation (2) offers a representative perspective of a fusion reaction. Note that the mass of the fusion reactants and products are considerable less than the fission reaction. However, large scale and controllable fusion is a scientific aspiration pending fruition. Nuclear fusion generally requires a preliminary fission event of an extraordinary scale. The pending thermonuclear yield from uncontrolled nuclear fusion transcends the yield of fission-only strategies [11].

Localized fusion catalyzed by antimatter offers a realistic pathway to a more 
controlled form of fusion propulsion. The current strategy involves the application of antiproton derived antimatter. This conceptual perspective has manifested in Ion Compressed Antiproton Nuclear (ICAN) system, which are conceptualized as ICAN and ICAN-II [12] [13]. However, storage and production of antiproton derived antimatter are debatable in terms of feasibility.

The application of positron antimatter derived from an ultra-intense laser incident on a high atomic number target, such as gold, offers the potential for remote generation of positron antimatter and the associated ultra-intense laser and energy source. As opposed to the requirement of an antimatter storage system, the positron antimatter can be generated on demand. Multiple mechanism pathways are available for antimatter catalyzed fusion. The objective of the research is to elucidate position antimatter catalyzed fusion by means of an ultra-intense laser through fundamental performance analysis.

\section{Preliminary Mission Concepts Incorporating Antimatter Initiated Fusion Propulsion}

ICAN (Ion Compressed Antiproton Nuclear) utilizes Antimatter Catalyzed Micro-Fission/Fusion (ACMF), which is envisioned for interplanetary space missions, such as Mars. Similar to Project Orion the ICAN utilizes a thruster shell as an evolution to the pusher plate for capturing thrust from a micro-thermonuclear detonation. The nuclear reaction pathway first involves a beam of antiprotons being accelerated to a target pellet from a storage device. The pellet would consist of uranium and hydrogen of sufficient quantities to evoke antimatter induced fission with subsequent fusion [12] [13].

Further analytical evolution of the role of ACMF has derived ICAN-II. The performance parameters for such a mission to Mars correspond to a specific impulse of approximately 13500 seconds and $100 \mathrm{kN}$ of thrust. Performance projections correspond to $140 \mathrm{ng}$ of antiprotons, which is equivalent to $10^{17}$ antiprotons. Such a degree of antiproton antimatter production would be on the order of approximately one year of dedicated antimatter production from resources, such as Fermilab. The mass estimations of ICAN-II are on the scale of in excess of 700 metric tons [13].

Although ICAN-II provides considerable insight as to the future role of antimatter initiated types of propulsion, there are many barriers to the near term operational likelihood of the concept. The storage of large quantities of antiprotons is not only a challenge by the shear economic magnitude, but also the safety of antiproton storage and transport to a space environment constitutes considerable risk. Furthermore the capacity to transport the supporting hardware to a preliminary location is space orbit is a topic of concern.

Similar to the proposal of Project New Orion as a photofission derived test and evaluation feasible alternative contrary to the thermonuclear proportion of Project Orion, the conceptual non-chemical propulsion endeavor advocates positron derived antimatter production for eliciting a micro-scale nuclear fusion event. Such a perspective is envisioned as more realistic for near-term testing 
and evaluation. The preliminary concept is elucidated through fundamental performance analysis and identification of a suitable mechanism for positron antimatter induced fusion.

\section{Fundamentals of Antimatter Induced Fusion}

The foundational geometry for antimatter initiated fusion is a pellet consisting of materials amenable to nuclear fusion. These materials are arranged in a concentric manner. The antimatter incident on the pellet is sufficient to commence nuclear fusion [14] [15] [16].

The characteristics of the fusion reaction can be a function of the selected materials for pending nuclear fusion. One configuration involves concentric spherical layers of $\mathrm{Li}_{2} \mathrm{DT}$. About the center of the pellet core would exist a sufficient quantity of antiprotons antimatter in suspended state. An implosion mechanism, such as through a chemical explosion, would commence antimatter-matter contact and pending annihilation. The resulting annihilation would generate a sufficient thermonuclear burn-wave throughout the majority of the $\mathrm{Li}_{2} \mathrm{DT}$ fuel for fusion [14].

Antimatter Catalyzed Micro-Fission/Fusion can be produced by a Uranium Deuterium-Tritium core. This core is encapsulated by gold representing a pusher. A key to this nuclear reaction pathway is the use of antimatter antiprotons to induce fission of the uranium from the interaction with antiprotons. Conventional uranium fission produces approximately $2-3$ neutrons for each fission reaction. However for an antiproton triggered fission of the uranium yields on the scale of 16 neutrons per fission event. This mechanism produces a high efficiency fusion burn [15].

Alternative configurations for the Antimatter Catalyzed Micro-Fission/Fusion concept exist, such as the material selection of the encompassing shell. A primary role of the shell is to provide mass for the temporary containment of the fusion fuel, so that for example $5 \%-10 \%$ of the fusion fuel can react. A preliminary uranium-238 shell with a surrounding Tungsten shell has been proposed. Additional fusion neutrons incident on the uranium-238 could further advance the fusion compression [16] [17].

There are two antimatter induced fusion mechanisms to be further investigated:

1) Antiproton volumetric ignition

2) Antiproton hotspot ignition [18]

Antiproton volumetric ignition consists of Deuterium-Tritium that is seeded with a heavy metal, such as uranium. The antimatter kinetic energy is adjusted to deposit in the fuel. The antimatter annihilation with the uranium seed yields short range super-fission. The resultant nuclear yield is the combination of the antiproton annihilation, Deuterium-Tritium fusion, and uranium fission [18].

Antiproton hotspot ignition consists of a Deuterium-Tritium core. Peripheral to the core is a concentric layer of Deuterium-Tritium interspersed with a heavy metal seed, such as uranium. The heavy metal seed is positioned in the ablator 
not the fusion fuel proximal to the pellet core. An advantage of this scenario is the clean Deuterium-Tritium fusion. The heavy metal only facilitates the ablator section [18].

A preliminary performance analysis for the scope of pulsed space propulsion has been conducted for antiproton volumetric ignition and antiproton hotspot ignition. The performance analysis estimates the resultant rocket exhaust velocity. The antiproton volumetric ignition scheme requires $6.5 \times 10^{17}$ antiprotons per target, which provides an exhaust velocity of $3.8 \times 10^{6} \mathrm{~m} / \mathrm{s}$. The antiproton hotspot ignition strategy requires $1.9 \times 10^{16}$ antiprotons per target, which produces an exhaust velocity corresponding to $8 \times 10^{6} \mathrm{~m} / \mathrm{s}$. The antiproton hotspot ignition strategy is selected and produces a greater exhaust velocity, while requiring a lower number of antiprotons per target [18].

\section{Positron Antimatter Derived by Ultra-Intense Laser to Initiate Fusion}

LeMoyne and Mastroianni during 2014 disclosed a pulsed space propulsion concept incorporating positron antimatter derived by an ultra-intense laser. The antimatter propulsion system was based on the observation of Lawrence Livermore National Laboratory successfully generating positron antimatter through applying an ultra-intense laser incident on a gold target to generate antimatter [2]. The successful disclosure of pulsed space propulsion incorporating positron antimatter through the operation of an ultra-intense laser, establishes the basis for the presentation of positron antimatter initiated fusion pulsed space propulsion.

The pulsed space propulsion concept incorporates the fusion ignition of pellets suitable for producing a nuclear fusion reaction. The mechanism for the antiproton hotspot ignition will be applied with positron antimatter substituting antiprotons. The pellets will consist of a Deuterium-Tritium core with a peripheral concentric layer of Deuterium-Tritium and a suitable heavy metal, such as uranium, to serve the function as an ablator. The positron antimatter will be generated on demand through an ultra-intense laser incident on a gold target positioned at the periphery of the pellet.

A notable benefit to this described concept is the alleviation of an antimatter storage system. The positrons could be generated on demand as a function of activating the ultra-intense laser. Therefore mission complexities, such as antimatter storage and safety of antimatter transportation, are ameliorated.

Furthermore, the inherent nature of a laser beam decouples the propulsion system from the energy source. For example, if the proposed positron initiated fusion pulsed space propulsion system was applied to a Earth to Moon application for the transport of bulk materials to a lunar outpost; the ultra-intense laser could be positioned on the Earth, space station, or the Moon. Basically the energy source for generating the ultra-intense laser beam to generate the positron antimatter could be far remote from the positron initiated fusion pulsed space propulsion system. The use of an ultra-intense laser serves a critical link for the 
generation of positron antimatter.

\section{Ultra-Intense Laser for Generating Positron Antimatter}

The conceptual approach for generating positrons through a laser on the order of $10^{20} \mathrm{~W} / \mathrm{cm}^{2}$ was derived by Shearer et al. in the early 1970 's [19]. With progressively diligent research, development, testing, and evaluation Lawrence Livermore National Laboratory generated positron antimatter through an ultraintense laser incident on a high atomic number target, such as gold. Approximately $90 \%$ of the positrons are emitted aft relative to the laser beam incident on the target. The selected target consisted of gold and approximately $1 \mathrm{~mm}$ in thickness [20] [21]. The ultra-intense laser pulse caused interaction between electron cloud and nuclei resulting in the generation of positron antimatter [20] [21] [22].

The mechanism for generating positron antimatter through an ultra-intense laser is the result of the Bethe-Heitler process. During the Bethe-Heitler process fast electrons generate high-energy bremsstrahlung photons that interact with the nuclei to produce positron-electron pairs as illustrated in Equations (3) and (4):

$$
\begin{aligned}
& e-+Z \rightarrow \gamma+e-Z \\
& \gamma+Z \rightarrow e++e-+Z
\end{aligned}
$$

e+: Positron

$e-$ Electron

$Z$ : High atomic number nuclei(such as gold)

$\gamma$. Bremsstrahlung photons [20] [22]

The Bethe-Heitler process is predominant for gold targets with a thickness of approximately $1 \mathrm{~mm}[22]$.

\section{Fundamental Performance Analysis of Positron Initiated Fusion Pulsed Space Propulsion through the Application of an Ultra-Intense Laser}

In consideration of the capabilities of the Lawrence Livermore National Laboratory Titan laser, which is on the $10^{20} \mathrm{~W} / \mathrm{cm}^{2}$ ultra-intense scale, $2 \times 10^{10}$ positrons can be generated with a roughly 1 ps pulse with laser beam incidence on an approximately $1 \mathrm{~mm}$ thick gold target. On the order of $90 \%$ of the generated positrons are discharged anisotropic and aft of the incidence laser beam to the gold target. The kinetic energy of the positrons is about $2 \mathrm{MeV}$. Therefore, each pulse generates $1.8 \times 10^{10}$ positrons per 1 ps pulse for the positron initiated fusion pulsed space propulsion application [20] [21].

The positron and electron pair comprises a rest mass of $2 \mathrm{mc}^{2}=1.02 \mathrm{MeV}$. Positrons and electrons have the equivalent rest mass $(m)$, and $(c)$ is the speed of light. Kinetic energy is conserved as kinetic energy is assumed to be elastic [10] [23]. With a positron energy of $2 \mathrm{MeV}$, the energy of each positron-electron annihilation event is derived to be $3.02 \mathrm{MeV}$ [2].

The rest mass of a proton and correlated antiproton is $938 \mathrm{MeV}$, so the proton-antiproton annihilation would be on the order of $1876 \mathrm{MeV}$ [10]. Approx- 
imately 621 positrons would represent the equivalent energy yield of an antiproton antimatter annihilation event. Therefore, $1.2 \times 10^{19}$ positrons per gold target would initiate positron induced fusion through the antiproton hotspot ignition mechanism of a pellet using a Deuterium-Tritium core associated with a peripheralconcentric layer consisting of Deuterium-Tritium integrated with a heavy metal seed, such as uranium, and gold about the outer layer of the pellet. Using positrons as an alternative to antiprotons to satisfy the energy thresholds for the antiproton hotspot ignition strategy would require on the order of $6.7 \times$ $10^{8}$ pulses from an ultra-intense laser. A notable feature of the proposed positron initiated fusion pulsed space propulsion through the application of an ultra- intense laser is the observation that this application only requires roughly one order of magnitude more of ultra-intense laser pulses when contrasted to the original positron antimatter pulsed space propulsion system published by LeMoyne and Mastroianni [2].

A notable feature of the proposed positron initiated fusion pulsed space propulsion is the considerable propulsive exhaust velocity. The performance analysis assumes the resultant plasma generates the propulsive exhaust through expansion with an ideal nozzle, such as a magnetic nozzle. The derived exhaust velocity is $8 \times 10^{6} \mathrm{~m} / \mathrm{s}$ with respect to the antiproton hotspot ignition mechanism [18].

Since an ideal nozzle for expanding the propulsive flow is applied to the performance analysis, the exhaust pressure is assumed to approach the equivalent of the vacuum in space. Therefore the specific impulse is directly proportional to the acceleration of gravity as demonstrated in Equation (5):

$$
\begin{aligned}
& I_{s p}=\frac{F}{\dot{m} g}=\frac{\dot{m} v}{\dot{m} g}=\frac{v}{g} \\
& I_{s p}: \text { Specific Impulse (seconds) } \\
& F: \operatorname{Thrust}(\text { Netwons }) \\
& \dot{m}: \operatorname{Mass} \text { Flow }(\mathrm{kg} / \mathrm{s}) \\
& g: \operatorname{Gravity}\left(\mathrm{m} / \mathrm{s}^{2}\right) \\
& v: \operatorname{Velocity}(\mathrm{m} / \mathrm{s})
\end{aligned}
$$

The specific impulse for the positron initiated fusion pulsed space propulsion through the application of an ultra-intense laser is $8.2 \times 10^{5}$ seconds. The derived specific impulse of the positron initiated fusion pulsed space propulsion through the application of an ultra-intense laser using the antiproton hotspot ignition mechanism implies suitability for the transport of bulk materials in support of space missions.

\section{Conclusion}

A pulsed space propulsion system using position antimatter to induce Deuterium-Tritium fusion through an ultra-intense laser incident on a gold target has been successfully presented and elucidated through fundamental performance 
analysis. Previous applications advocating antimatter induced fusion have featured the role of antiproton antimatter. However many associated challenges, such as production, storage, and transport, impart considerable complexity. Positron antimatter can be derived from an ultra-intense laser incident on a high atomic number target through the Bethe-Heitler process. As previously demonstrated the use of ultra-intense lasers can decouple the laser and energy source from the in flight generation of positron antimatter. With a review of the means for deriving antimatter induced fusion the antiproton hotspot ignition mechanism was selected. The mechanism encompasses a pellet consisting of a DeuteriumTritium core and a peripheral concentric layer of Deuterium-Tritium and a suitable heavy metal, such as uranium, which functions as an ablator. Positrons are generated by ultra-intense laser incident on a gold target about the periphery of the pellet. Fundamental performance analysis derives a considerable specific impulse, which implies mission suitability of the proposed propulsion system for transport of bulk materials to support space missions.

\section{References}

[1] LeMoyne, R. (2012) Fundamental Architecture and Analysis of Ramjet Propulsion System Utilizing In-Situ Laser Derived Antimatter. Proceedings of 18 th AIAA/3 AF International Space Planes and Hypersonic Systems and Technologies Conference, Tours, September 2012. https://doi.org/10.2514/6.2012-5863

[2] LeMoyne, R. and Mastroianni, T. (2014) Fundamental Architecture and Analysis of an Antimatter Ultra-Intense Laser Derived Pulsed Space Propulsion System. Journal of Applied Mathematics and Physics, 2, 10-18. https://doi.org/10.4236/jamp.2014.25002

[3] LeMoyne, R. and Mastroianni, T. (2014) Advanced Concept Ramjet Propulsion System Utilizing In-Situ Positron Antimatter Derived from Ultra-Intense Laser with Fundamental Performance Analysis. Journal of Applied Mathematics and Physics, 2, 19-26. https://doi.org/10.4236/jamp.2014.25003

[4] LeMoyne, R. and Mastroianni, T. (2014) Fundamental Architecture and Analysis of an Antimatter Ultra-Intense Laser Derived Combined Cycle Ramjet-Rocket Propulsion System. Proceedings of 19 th AIAA International Space Planes and Hypersonic Systems and Technologies Conference, Atlanta, June 2014. https://doi.org/10.2514/6.2014-2789

[5] LeMoyne, R. and Mastroianni, T. (2015) Fundamental Architecture and Performance Analysis of Photofission Pulsed Space Propulsion System Using Ultra-Intense Laser. Journal of Applied Mathematics and Physics, 3, 436-442. https://doi.org/10.4236/jamp.2015.34055

[6] LeMoyne, R. and Mastroianni, T. (2016) Project New Orion: Pulsed Nuclear Space Propulsion Using Photofission Activated by Ultra-Intense Laser. Journal of Applied Mathematics and Physics, 4, 630-636. https://doi.org/10.4236/jamp.2016.44071

[7] Nance, J.C. (1965) Nuclear Pulse Propulsion. IEEE Transactions on Nuclear Science, 12, 177-182. https://doi.org/10.1109/TNS.1965.4323511

[8] Dyson, F.J. (1968) Interstellar Transport. Physics Today, 21, 41-45. https://doi.org/10.1063/1.3034534

[9] Schmidt, G.R., Bunometti, J.A. and Morton, P.J. (2000) Nuclear Pulse Propulsion-Orion and beyond. Proceedings of 36 th AIAMI ASME/SAE/ ASEE Joint Pro- 
pulsion Conference \& Exhibition, Huntsville, July 2000. https://doi.org/10.2514/6.2000-3856

[10] Serway, R.A., Moses, C.J. and Moyer, C.A. (1989) Modern Physics. Saunders College, Philadelphia.

[11] Loeber, C.R. (2005) Building the Bombs: A History of the Nuclear Weapons Complex. Sandia National Laboratories, Albuquerque.

[12] Chiang, P.R., Lewis, R.A., Smith, G.A., Gazze, C., Higman, K., Newton, R., Chiaverini, M., Dailey, J., Surratt, M., Werthman, W.L., Chakrabarti, S. and Cracraft, P. (1993) An Antiproton Driver for ICF Propulsion. NASA Propulsion Engineering Research Center, 1, 203-206.

[13] Gaidos, G., Laiho, J., Lewis, R.A., Smith, G.A., Dundore, B., Fulmer, J. and Chakrabarti, S. (1998) Antiproton-Catalyzed Microfission/Fusion Propulsion Systems for Exploration of the Outer Solar System and Beyond. AIP Conference Proceedings, 420, 1365-1372.

[14] Gsponer, A. and Hurni, J.P. (2005) Antimatter Induced Fusion and Thermonuclear Explosions. arXiv:preprint physics/0507125v2. https://arxiv.org/abs/physics/0507125v2

[15] Frisbee, R.H., Brophy, J.R. and Van Landingham, E.E. (1996) Status of NASA's Advanced Propulsion Technology Program. Proceedings of 32nd AIAA, ASME, SAE, and ASEE, Joint Propulsion Conference and Exhibit, Lake Buena Vista, July 1996. https://doi.org/10.2514/6.1996-2784

[16] Cassenti, B.N. and Kammash, T. (2009) Future of Antiproton Triggered Fusion Propulsion. Proceedings of 45 th AIAA/ASME/SAE/ ASEE Joint Propulsion Conference \& Exhibit, Denver, August 2009. https://doi.org/10.2514/6.2009-4871

[17] Cassenti, B.N., Kammash, T. and Galbraith, D.L. (1996) Antiproton Catalyzed Fusion Propulsion for Interplanetary Missions. Proceedings of 32nd AIAA, ASME, $S A E$, and ASEE, Joint Propulsion Conference and Exhibit, Lake Buena Vista, July 1996. https://doi.org/10.2514/6.1996-3068

[18] Perkins, L.J., Orth, C.D. and Tabak, M. (2003) On the Utility of Antiprotons as Drivers for Inertial Confinement Fusion, Lawrence Livermore National Laboratory. Department of Energy, 1-49.

[19] Shearer, J.W., Garrison, J., Wong, J. and Swain, J.E. (1973) Pair Production by Relativistic Electrons from an Intense Laser Focus. Physical Review A, 8, 1582-1588. https://doi.org/10.1103/PhysRevA.8.1582

[20] Chen, H., Wilks, S.C., Bonlie, J.D., Chen, S.N., Cone, K.V., Elberson, L.N., Gregori, G., Meyerhofer, D.D., Myatt, J., Price, D.F., Schneider, M.B., Shepherd, R., Stafford, D.C., Tommasini, R., Van Maren, R. and Beiersdorfer, P. (2009) Making Relativistic Positrons Using Ultraintense Short Pulse Lasers. Physics of Plasmas, 16. https://doi.org/10.1063/1.3271355

[21] Chen, H., Wilks, S.C., Bonlie, J.D., Liang, E.P., Myatt, J., Price, D.F., Meyerhofer, D.D. and Beiersdorfer, P. (2009) Relativistic Positron Creation Using Ultraintense Short Pulse Lasers. Physical Review Letters, 102. https://doi.org/10.1103/physrevlett.102.105001

[22] Nakashima, K. and Takabe, H. (2002) Numerical Study of Pair Creation by Ultraintense Lasers. Physics of Plasmas, 9, 1505-1512. https://doi.org/10.1063/1.1464145

[23] Serway, R.A. (1990) Physics for Scientists and Engineers. Saunders College, Philadelphia.

[24] Sutton, G.P. (1992) Rocket Propulsion Elements. Wiley-Interscience, New York. 
Submit or recommend next manuscript to SCIRP and we will provide best service for you:

Accepting pre-submission inquiries through Email, Facebook, LinkedIn, Twitter, etc. A wide selection of journals (inclusive of 9 subjects, more than 200 journals)

Providing 24-hour high-quality service

User-friendly online submission system

Fair and swift peer-review system

Efficient typesetting and proofreading procedure

Display of the result of downloads and visits, as well as the number of cited articles Maximum dissemination of your research work

Submit your manuscript at: http://papersubmission.scirp.org/

Or contact jamp@scirp.org 